\title{
A retrospective database analysis of insulin use patterns in insulin-naïve patients with type 2 diabetes initiating basal insulin or mixtures
}

This article was published in the following Dove Press journal:

Patient Preference and Adherence

23 June 2010

Number of times this article has been viewed

\author{
Machaon MK Bonafede' \\ Anupama Kalsekar ${ }^{2}$ \\ Manjiri Pawaskar ${ }^{2}$ \\ Kimberly M Ruiz \\ Amelito M Torres ${ }^{3}$ \\ Karen R Kelly ${ }^{2}$ \\ Suellen M Curkendall ${ }^{3}$ \\ 'Thomson Reuters Inc, Cambridge, \\ Massachusetts, USA; ${ }^{2}$ Eli Lilly and \\ Company, Indianapolis, Indiana, USA; \\ ${ }^{3}$ Thomson Reuters Inc, Washington, \\ DC, USA
}

Objective: To describe insulin persistence among patients with type 2 diabetes initiating insulin therapy with basal insulin or insulin mixtures and determine factors associated with nonpersistence.

Research design and methods: The Thomson Reuters MarketScan ${ }^{\circledR}$ databases were used to retrospectively analyze insulin-naïve patients with type 2 diabetes by initiating insulin therapy. Insulin use was described using a variety of measures. The persistence to insulin was described using both a gap-based measure and the number of claims measure.

Results: Patients in the basal insulin cohort $(\mathrm{N}=15,255)$ primarily used insulin analogs $(88.1 \%)$ and vial and syringe (97\%). Patients in the mixture cohort $(\mathrm{N}=2,732)$ were more likely to initiate on human insulin mixtures (62.5\%) and vial and syringe (68.1\%). Average time between insulin refills was 80 and 71 days for basal and mixture initiators, respectively. Nearly, $75 \%$ of basal insulin initiators and $65 \%$ of insulin mixture initiators had a 90 -day gap in insulin prescriptions. More than half of all the patients had at least one insulin prescription per quarter. Patients initiating with insulin analogs were more likely to be persistent compared with those initiating with human insulin across both cohorts and measures of persistence $(P<0.001)$.

Conclusion: Persistence to insulin therapy is poorer than one would anticipate, but appears to be higher in users of insulin analogs and insulin mixtures.

Keywords: insulin persistence, basal insulin, insulin mixtures

\section{Introduction}

Type 2 diabetes is a common and expensive disease, with 17.5 million diagnosed cases and $\$ 174$ billion in acute-care costs in the United States in 2007. ${ }^{1}$ The goal of type 2 diabetes treatment is to maintain a glycosylated hemoglobin (HbAlc) of $<7 \%{ }^{2}$ To meet this goal, many patients with type 2 diabetes eventually require insulin therapy to maintain glycemic control because of progressive $\beta$-cell dysfunction. Health care providers and patients are often reluctant to initiate insulin therapy, as this therapy is intended to be a permanent change and requires strict adherence and persistence to diet and medication compared with oral antihyperglycemic agents. A significant percent of patients with diabetes experiences difficulty in taking insulin as prescribed by their doctor. For example, in a review article, $\mathrm{Cramer}^{3}$ reported that persistence to insulin varies but is often quite low and three reviewed studies reported that $16 \%-49 \%$ of patients are persistent at 6-12 months. ${ }^{4-6}$ Research also suggests that factors related to injection, ${ }^{7}$ complexity of the medication regimen, ${ }^{3}$ and higher frequency dosing schedules ${ }^{8,9}$ are key barriers to insulin therapy. Thus, several treatment-related factors may be significant barriers to successful long-term treatment for some patients.
Correspondence: Machaon MK Bonafede Thomson Reuters Inc, 37 Lowell Street, Andover MA 0I8I0, USA

Tel $+\mathrm{I}-585-766-8622$

Fax + I-617-492-9365

Email machaon.bonafede@

thomsonreuters.com 
The primary objective of this study was to describe the persistence to insulin among insulin-naïve patients with type 2 diabetes, who initiated insulin therapy with basal (long-acting) insulin or mixtures. The secondary objective was to determine risk factors for poor persistence with insulin therapy.

\section{Research design and methods}

This study used a retrospective approach using administrative claims data. Two MarketScan ${ }^{\circledR}$ research databases from Thomson Reuters were used in this study: the Commercial Claims and Encounters (Commercial) and the Medicare Supplemental and Coordination of Benefits (Medicare Supplemental and COB) databases. The Commercial database contains the details of inpatient, outpatient, emergency room (ER), and outpatient prescription drug experience of several million individuals and their dependents (annually), who were covered under a variety of fee-for-service and capitated health plans, including exclusive provider organizations, preferred provider organizations, point of service plans, indemnity plans, and health maintenance organizations. The overall database includes individuals from over 100 self-insured large employers and health plans. The Medicare
Supplemental and COB database contains the healthcare experience of individuals with Medicare Supplemental insurance paid for by employers. Because it covers an older population than does the Commercial database, the Medicare Supplemental database is a key data source. Both the Medicare-covered portion of payment (represented as $\mathrm{COB}$ amount or $\mathrm{COB}$ ) and the employer-paid portion are included in this database.

\section{Inclusion criteria}

Patients had to be new insulin initiators (basal or mixtures) between July 1, 2001, and December 31, 2006 (index date). Table 1 provides a list of insulins included in this study, which are classified by types: human vs analog and basal vs mixture. A 6-month preperiod was used to establish new insulin use, a diagnosis of type 2 diabetes (identified by ICD-9-CM codes), and the presence of stable diabetic therapy, as evidenced by having at least two prescriptions for exenatide $\left(\right.$ Byetta $^{\circledR}$ ) or an oral antidiabetic agent; the two oral antidiabetic agents could be for the same or different agents; patients were not required to use exenatide or oral antidiabetic agents in the postindex period. Subjects had to be enrolled in a qualified health plan with concurrent continuous pharmacy enrollment

Table I Insulin types and examples

\begin{tabular}{|c|c|c|c|}
\hline Class & Generic name & Brand name & Type \\
\hline Basal & $\begin{array}{l}\text { Insulin human isophane } \\
\text { (NPH) }\end{array}$ & $\begin{array}{l}\text { Humulin N; } \\
\text { Novolin N; } \\
\text { Relion/Novolin N; } \\
\text { Insulatard }\end{array}$ & Human \\
\hline Basal & $\begin{array}{l}\text { Insulin human zinc } \\
\text { (Lente) }\end{array}$ & $\begin{array}{l}\text { Humulin L; } \\
\text { Novolin L }\end{array}$ & Human \\
\hline Basal & $\begin{array}{l}\text { Insulin human zinc, } \\
\text { extended (Ultralente) }\end{array}$ & Humulin U & Human \\
\hline Basal & Insulin detemir & Levemir & Analog \\
\hline Basal & $\begin{array}{l}\text { Insulin glargine, } \\
\text { Recombinant }\end{array}$ & Lantus & Analog \\
\hline Insulin mixtures & $\begin{array}{l}\text { Insulin human isophane } \\
(\mathrm{NPH}) / \text { Insulin human regular }\end{array}$ & $\begin{array}{l}\text { Humulin } 50 / 50 \\
\text { Humulin } 70 / 30\end{array}$ & Human \\
\hline Insulin mixtures & $\begin{array}{l}\text { Insulin human isophane } \\
(\mathrm{NPH}) / \text { Insulin human regular }\end{array}$ & $\begin{array}{l}\text { Novolin 70/30; } \\
\text { Relion/Novolin 70/30 }\end{array}$ & Human \\
\hline Insulin mixtures & $\begin{array}{l}\text { Insulin lispro/ } \\
\text { Insulin lispro protamine }\end{array}$ & $\begin{array}{l}\text { Humalog mix } 50 / 50 \\
\text { Humalog } \operatorname{mix} 75 / 25\end{array}$ & Analog \\
\hline Insulin mixtures & $\begin{array}{l}\text { Insulin aspart/ } \\
\text { Insulin aspart protamine }\end{array}$ & Novolog mix 70/30 & Analog \\
\hline Mealtime & Insulin aspart, recombinant & Novolog & Analog \\
\hline Mealtime & Insulin glulisine & Apidra & Analog \\
\hline Mealtime & Insulin lispro, recombinant & Humalog; lispro-PFC & Analog \\
\hline Mealtime & Insulin human regular & $\begin{array}{l}\text { Humulin R; } \\
\text { Novolin R; } \\
\text { Relion/Novolin R }\end{array}$ & Human \\
\hline Mealtime & $\begin{array}{l}\text { Insulin human regular, } \\
\text { buffered }\end{array}$ & $\begin{array}{l}\text { Humulin BR; } \\
\text { Velosulin BR }\end{array}$ & Human \\
\hline
\end{tabular}


for a 21-month period spanning the 6-month preindex period through the 15-month study period between January 1, 2001, and March 31, 2008. A moving target index data approach was used to maximize sample size and minimize the impact of seasonal effects (such as annual caps) on utilization.

\section{Exclusion criteria}

Patients were excluded if they had evidence of gestational diabetes (ICD-9-CM code of 648.8x), had evidence of type 1 diabetes (ICD-9-CM code of 250.x1 or 250.x3 or DRG 295), or used inhaled insulin or an insulin pump anytime in the observation period. In order to create a more homogenous patient population of insulin initiators, patients were also excluded if they used mealtime (short-acting) insulin or both basal and mixed insulins in the postindex period. Patients with only one insulin prescription claim were also excluded from this analysis.

\section{Insulin use and variables of interest}

Index insulin characteristics such as insulin type (human vs analog) and administration (pen vs vial and syringe) were noted.

Several study variables were used to describe insulin use. These include the following:

1. Number of prescription claims for index insulin at 3,6 , and 12 months

2. Average daily insulin supply calculated at 3,6, and 12 months postindex date by calculating the total insulin supply in $\mathrm{mL}$ dispensed and dividing by the number of days in that period

3. Average time between refills of index insulin

4. Proportion that switched to a different insulin type or a different insulin administration.

\section{Insulin persistence measures}

Daily adherence to insulin, defined as, "the extent to which a patient acts in accordance with the prescribed interval and dose of a dosing regimen," was of interest but could not be determined from a database of prescription claims. ${ }^{10}$ This study instead focused on evaluating persistence at 12 months, defined as the time from therapy initiation to discontinuation, including a limit on the days between fills. ${ }^{10}$ Persistence was evaluated at 12 months by the absence of gaps between refills (measure 1) or the number of refills within a prespecified period (measure 2). Under measure 1, patients were considered persistent at 3 months if they did not have a 90-day gap in index insulin prescriptions, which started prior to the end of the third month. Persistence at 6 and 12 months were defined similarly but relative to the end of the sixth and twelfth months, respectively. Under measure 2, patients were considered persistent at 3 months if they had two index insulin prescriptions in the first 4 months. Patients were considered persistent at 6 months if they had at least three claims in the first 7 months, including at least one in 1-3 months and at least one claim in 4-6 months. Patients were considered persistent at 12 months if they had at least four insulin prescriptions in 12 months following their index prescription, with at least one index insulin prescription in each quarter. Measure 1 represents a standard definition of persistence, ${ }^{10}$ and measure 2 is a more lenient measure and hence was included as a sensitivity analysis. Nonpersistence, as determined by these measures, does not imply a permanent discontinuation from insulin and may be a marker for intermittent use.

Persistent and nonpersistent patients were compared using standard tests of statistical significance. Chi-square tests were used to evaluate the statistical significance of differences for categorical variables; however, two-tailed $t$-tests and analysis of variance were used for continuous variables. A series of logistic regression models were used to examine factors associated with being persistent with insulin therapy at 12 months. Factors included age, gender, location, type of insurance, insulin type, insulin administration mode, Deyo Charlson Comorbidity Index (described by Deyo et $\mathrm{al}^{11}$ ), presence of hospitalization, presence of ER visit, presence of diabetes complications, presence of macrovascular complications, presence of mental health disorders, count of other diabetes medications, and average copayment per prescription of insulin. An alpha of 0.05 was used for all analyses. Due to the larger sample size, a power calculation was not performed.

\section{Results}

Of the 505,898 patients with a index prescription of basal or mixture insulins, 69,318 met the inclusion criteria, of which 17,987 patients also met the exclusion criteria and formed the final sample for this analysis. Nearly, $85 \%$ of these patients $(\mathrm{N}=15,255)$ initiated insulin therapy with basal insulin using insulin analogs $(88.1 \%)$ and vial and syringe (97\%) (Table 2). Less than 1\% of the basal insulin initiators switched to a different insulin type (analog or human) or different insulin administration mode in the 12 months following index insulin prescription. Conversely, in the insulin mixture cohort $(\mathrm{N}=2,732)$, most patients initiated insulin using human insulin mixtures $(62.5 \%)$ and vial and syringe (68.1\%). Approximately, $7 \%$ of patients switched to a different insulin type and insulin administration mode in the 12 months following index insulin prescription. 
Table 2 Insulin use

\begin{tabular}{|c|c|c|c|c|c|c|}
\hline & \multicolumn{3}{|c|}{ Basal insulin $(N=\mid 5,255)$} & \multicolumn{3}{|c|}{ Insulin mixtures $(\mathbf{N}=\mathbf{2 , 7 3 2})$} \\
\hline & Mean & Median & IQ range & Mean & Median & IQ range \\
\hline \multicolumn{7}{|c|}{ No. of claims } \\
\hline $3 \mathrm{mo}$ & 2.07 & 2 & $(I, 3)$ & 2.42 & 2 & $(I, 3)$ \\
\hline $6 \mathrm{mo}$ & 3.40 & 3 & $(2,4)$ & 3.97 & 4 & $(2,5)$ \\
\hline $12 \mathrm{mo}$ & 5.99 & 5 & $(4,8)$ & 6.93 & 6 & $(4,9)$ \\
\hline \multicolumn{7}{|c|}{ Quantity per day (mL) } \\
\hline $3 \mathrm{mo}$ & 0.25 & 0.22 & $(0.17,0.33)$ & 0.31 & 0.33 & $(0.22,0.33)$ \\
\hline $6 \mathrm{mo}$ & 0.20 & 0.17 & $(0.11,0.25)$ & 0.26 & 0.22 & $(0.17,0.33)$ \\
\hline $12 \mathrm{mo}$ & 0.18 & 0.16 & $(0.11,0.22)$ & 0.22 & 0.19 & $(0.12,0.30)$ \\
\hline \multicolumn{7}{|c|}{ Time between refills (days) } \\
\hline $12 \mathrm{mo}$ & 80.11 & 67.40 & $(45.20,95.67)$ & 71.32 & 55.50 & $(27.92,84.00)$ \\
\hline \multicolumn{7}{|c|}{ Time until change in insulin administration (days): pen vs vial and syringe } \\
\hline $12 \mathrm{mo}$ & 192.80 & 189 & $(97,286)$ & $|26.7|$ & 92.5 & $(22.5,224.5)$ \\
\hline \multicolumn{7}{|c|}{ Time until change in insulin type (days): human vs analog } \\
\hline $12 \mathrm{mo}$ & 164.03 & 159 & $(66,257)$ & 139.87 & 118 & $(49,225)$ \\
\hline
\end{tabular}

Basal insulin initators used an average of two other antidiabetic medications in the follow-up period compared with an average of 1.4 for insulin mixture initiators. Both basal and insulin mixture initiators had similar prevalence of diabetes complications ( $22 \%$ and $24 \%$, respectively), macrovascular complications ( $49 \%$ and $51 \%$, respectively), and mental health disorders (5\% and 6.5\%, respectively). Mean Deyo Charlson Comorbidity Index scores were also similar (1.9 for basal insulin initiators and 2.1 for insulin mixture initiators).

As shown in Table 2, the mean number of claims for basal and mixture insulins increased over time, but the average daily supply decreased. For eg, basal insulin index patients filled an average of 2.07 prescriptions in the first 3 months, 3.4 prescriptions in the first 6 months, and 5.99 in the whole year of the study, which corresponds to 0.69 prescription fills per month in the first 3 months and 0.57 and 0.50 prescription fills per month in the subsequent time periods. The average daily supply follows a similar, albeit less pronounced trend over the same time periods, dropping from $0.25 \mathrm{~mL}$ ( $25 \mathrm{IU}$ ) per day to $0.20 \mathrm{~mL}$ (20 IU) per day and eventually to $0.18 \mathrm{~mL}$ (18 IU) per day. The average time between refills over 12 months was slightly higher among basal index patients (mean: 80.1 days, median: 67.4 days) than mixture index patients (mean: 71.3 days, median: 55.5 days).

Using measure 1, persistence in the basal cohort was $50.6 \%, 38.5 \%$, and $26.5 \%$; and persistence in the mixture cohort was $58.5 \%, 47.6 \%$, and $35.0 \%$ at 3,6 , and 12 months, respectively. In other words, almost three-quarters (73.5\%) of the patients who initiated insulin therapy with basal insulin had a 90-day gap between prescriptions at some point during the study period. Similarly, more than half of the mixture index patients (65.0\%) had a 90-day gap between prescriptions. The start of a 90-day gap was, on average, 80 days and 90.6 days for basal insulin and insulin mixture initiators, respectively, after their index insulin prescription. Among basal insulin initiators, $38.4 \%$ had a 90 -day gap immediately after their index prescription, and $6.7 \%$ had a 90-day gap that start within 30 days of their index prescription. Among insulin mixture initiators, $32.2 \%$ had a 90 -day gap immediately after their index prescription, and $7.2 \%$ had a 90-day gap that start within 30 days of their index prescription. The majority of patients with a 90 -day gap had another prescription following the period; $86.9 \%$ of basal index patients and $81.5 \%$ of mixture index patients restarted insulin following a 90-day gap. The time between the start of the 90-day gap and the next prescription was similar in both groups (basal index mean: 143.4 days, median: 120 days; mixture index mean: 145.9 days, median: 121 days).

Because measure 2 allowed for longer time between refills, more patients were likely to be classified as persistent with this measure. Using measure 2, persistence was $83.4 \%, 67.1 \%$, and $55.8 \%$ among basal insulin initiators and $86.7 \%, 71.0 \%$, and $59.1 \%$ among insulin mixture initiators at 3, 6, and 12 months, respectively. The agreement between measures 1 and 2 at 3, 6, and 12 months was $67 \%, 71 \%$, and $71 \%$ among basal insulin initiators and $72 \%, 77 \%$, and $76 \%$ among insulin mixture initiators, respectively. Agreement was measured as the number of concordant pairs divided by the number of discordant pairs.

Table 3 describes basal and mixture insulin initiators according to their persistence status determined by measure 2 . 
Table 3 Demographic and clinical characteristics

\begin{tabular}{|c|c|c|c|c|c|c|c|c|}
\hline & \multicolumn{4}{|c|}{ Basal insulin initiators } & \multicolumn{4}{|c|}{ Insulin mixture initiators } \\
\hline & $\begin{array}{l}\text { Persistent } \\
N=8,5 \text { I5 }\end{array}$ & $\begin{array}{l}\text { Nonpersistent } \\
N=6,740\end{array}$ & $P$ value & $\begin{array}{l}\text { Total } \\
N=15,255\end{array}$ & $\begin{array}{l}\text { Persistent } \\
N=1,615\end{array}$ & $\begin{array}{l}\text { Nonpersistent } \\
\mathbf{N}=1,1 \text { I } 7\end{array}$ & $P$ value & $\begin{array}{l}\text { Total } \\
N=2,732\end{array}$ \\
\hline & $\begin{array}{l}\text { Mean \% } \\
\text { (SD) }\end{array}$ & $\begin{array}{l}\text { Mean \% } \\
(\mathrm{SD})\end{array}$ & & $\begin{array}{l}\text { Mean \% } \\
\text { (SD) }\end{array}$ & $\begin{array}{l}\text { Mean \% } \\
(\text { SD) }\end{array}$ & $\begin{array}{l}\text { Mean \% } \\
(\text { SD) }\end{array}$ & & $\begin{array}{l}\text { Mean \% } \\
\text { (SD) }\end{array}$ \\
\hline Gender: Female & $43.43 \%$ & $45.43 \%$ & & $44.31 \%$ & $47.93 \%$ & $50.13 \%$ & & $48.83 \%$ \\
\hline Age (mean, SD) & $\begin{array}{l}59.44 \\
(11.38)\end{array}$ & $\begin{array}{l}60.01 \\
(12.17)\end{array}$ & 0.003 & $\begin{array}{l}59.69 \\
(11.74)\end{array}$ & $\begin{array}{l}60.67 \\
(11.89)\end{array}$ & $\begin{array}{l}59.87 \\
(12.49)\end{array}$ & 0.093 & $\begin{array}{l}60.34 \\
(12.14)\end{array}$ \\
\hline \multicolumn{9}{|l|}{ Age group } \\
\hline$<18$ & $0.07 \%$ & $0.06 \%$ & $<0.000$ I & $0.07 \%$ & $0.06 \%$ & $0.00 \%$ & 0.321 & $0.04 \%$ \\
\hline $18-34$ & $1.12 \%$ & $1.65 \%$ & & $1.35 \%$ & $1.49 \%$ & $1.97 \%$ & & $1.68 \%$ \\
\hline $35-44$ & $7.00 \%$ & $7.45 \%$ & & $7.20 \%$ & $6.69 \%$ & $7.97 \%$ & & $7.21 \%$ \\
\hline $45-54$ & $25.01 \%$ & $23.84 \%$ & & $24.50 \%$ & $21.98 \%$ & $24.26 \%$ & & $22.91 \%$ \\
\hline $55-64$ & $37.49 \%$ & $33.87 \%$ & & $35.89 \%$ & $33.37 \%$ & $31.87 \%$ & & $32.76 \%$ \\
\hline $65-74$ & $18.27 \%$ & $19.15 \%$ & & $18.66 \%$ & $22.66 \%$ & $20.14 \%$ & & $21.63 \%$ \\
\hline$\geq 75$ & $11.04 \%$ & $13.98 \%$ & & $12.34 \%$ & $13.75 \%$ & $13.79 \%$ & & $13.76 \%$ \\
\hline \multicolumn{9}{|l|}{ Location } \\
\hline Urban & $77.79 \%$ & $79.58 \%$ & 0.026 & $78.58 \%$ & $78.20 \%$ & $77.08 \%$ & 0.153 & $77.75 \%$ \\
\hline Rural & $21.90 \%$ & $20.10 \%$ & & $21.11 \%$ & $21.61 \%$ & $22.29 \%$ & & $21.89 \%$ \\
\hline Unknown & $0.31 \%$ & $0.31 \%$ & & $0.31 \%$ & $0.19 \%$ & $0.63 \%$ & & $0.37 \%$ \\
\hline \multicolumn{9}{|l|}{ Index insulin type } \\
\hline Human & $10.43 \%$ & $13.71 \%$ & $<0.0001$ & $11.88 \%$ & $59.20 \%$ & $67.23 \%$ & $<0.0001$ & $62.48 \%$ \\
\hline Analog & $89.57 \%$ & $86.29 \%$ & & $88.12 \%$ & $40.80 \%$ & $32.77 \%$ & & $37.52 \%$ \\
\hline \multicolumn{9}{|c|}{ Index insulin administration } \\
\hline Vials and syringes & $97.32 \%$ & $96.51 \%$ & 0.004 & $96.96 \%$ & $67.99 \%$ & $68.31 \%$ & 0.860 & $68.12 \%$ \\
\hline Pens & $2.68 \%$ & $3.49 \%$ & & $3.04 \%$ & $32.01 \%$ & $31.69 \%$ & & $31.88 \%$ \\
\hline \multicolumn{9}{|c|}{ Change in index insulin } \\
\hline $\begin{array}{l}\text { Human vs analog } \\
\text { change }\end{array}$ & $2.50 \%$ & $2.52 \%$ & 0.935 & $0.25 \%$ & $7.12 \%$ & $6.09 \%$ & 0.288 & $6.70 \%$ \\
\hline $\begin{array}{l}\text { Mean time to } \\
\text { change }\end{array}$ & $\begin{array}{l}159.25 \\
(105.94)\end{array}$ & $\begin{array}{l}170.02 \\
(\mid 12.85)\end{array}$ & 0.338 & $\begin{array}{l}164.03 \\
(109.05)\end{array}$ & $\begin{array}{l}145.43 \\
(104.95)\end{array}$ & $\begin{array}{l}130.47 \\
(106.70)\end{array}$ & 0.356 & $\begin{array}{l}139.87 \\
(105.56)\end{array}$ \\
\hline $\begin{array}{l}\text { Pen vs vial and } \\
\text { syringe change }\end{array}$ & $0.73 \%$ & $0.76 \%$ & 0.838 & $0.74 \%$ & $8.48 \%$ & $6.00 \%$ & 0.015 & $7.47 \%$ \\
\hline $\begin{array}{l}\text { Mean time to } \\
\text { change }\end{array}$ & $\begin{array}{l}185.82 \\
(106.88)\end{array}$ & $\begin{array}{l}201.27 \\
(106.99)\end{array}$ & 0.446 & $\begin{array}{l}192.80 \\
(106.73)\end{array}$ & $\begin{array}{l}135.69 \\
(115.19)\end{array}$ & $\begin{array}{l}108.34 \\
(103.73)\end{array}$ & 0.102 & $\begin{array}{l}|26.7| \\
(\mid 12.04)\end{array}$ \\
\hline \multicolumn{9}{|c|}{ Insulin use: 12 months } \\
\hline $\begin{array}{l}\text { No. of index } \\
\text { insulin claims }\end{array}$ & $\begin{array}{l}7.83 \\
(3.08)\end{array}$ & $\begin{array}{l}3.69 \\
(1.52)\end{array}$ & $<0.0001$ & $\begin{array}{l}6.00 \\
(3.25)\end{array}$ & $\begin{array}{l}9.13 \\
(3.63)\end{array}$ & $\begin{array}{l}3.74 \\
(1.88)\end{array}$ & $<0.0001$ & $\begin{array}{l}6.93 \\
(4.03)\end{array}$ \\
\hline $\begin{array}{l}\text { Index insulin } \\
\text { quantity per day }\end{array}$ & $\begin{array}{l}0.23 \\
(0.092)\end{array}$ & $\begin{array}{l}0.11 \\
(0.05)\end{array}$ & $<0.0001$ & $\begin{array}{l}0.18 \\
(0.10)\end{array}$ & $\begin{array}{l}0.30 \\
(0.13)\end{array}$ & $\begin{array}{l}0.12 \\
(0.07)\end{array}$ & $<0.000$ I & $\begin{array}{l}0.22 \\
(0.14)\end{array}$ \\
\hline $\begin{array}{l}\text { Time between } \\
\text { index insulin claims }\end{array}$ & $\begin{array}{l}57.95 \\
(22.99)\end{array}$ & $\begin{array}{l}108.11 \\
(67.41)\end{array}$ & $<0.0001$ & $\begin{array}{l}80.11 \\
(54.07)\end{array}$ & $\begin{array}{l}49.32 \\
(20.65)\end{array}$ & $\begin{array}{l}103.12 \\
(72.36)\end{array}$ & $<0.000$ I & $\begin{array}{l}71.32 \\
(55.60)\end{array}$ \\
\hline \multicolumn{9}{|c|}{ Concomitant medications } \\
\hline $\begin{array}{l}\text { Diabetes } \\
\text { medication count }\end{array}$ & $\begin{array}{l}2.00 \\
(0.96)\end{array}$ & $\begin{array}{l}2.12 \\
(1.00)\end{array}$ & $<0.0001$ & $\begin{array}{l}2.05 \\
(0.98)\end{array}$ & $\begin{array}{l}1.27 \\
(1.05)\end{array}$ & $\begin{array}{l}1.66 \\
(1.05)\end{array}$ & $<0.0001$ & $\begin{array}{l}1.43 \\
(1.06)\end{array}$ \\
\hline $\begin{array}{l}\text { Cardiovascular } \\
\text { medication count }\end{array}$ & $\begin{array}{l}3.8 I \\
(2.2 I)\end{array}$ & $\begin{array}{l}3.79 \\
(2.32)\end{array}$ & 0.496 & $\begin{array}{l}3.80 \\
(2.26)\end{array}$ & $\begin{array}{l}3.95 \\
(2.38)\end{array}$ & $\begin{array}{l}3.79 \\
(2.42)\end{array}$ & 0.076 & $\begin{array}{l}3.88 \\
(2.40)\end{array}$ \\
\hline \multicolumn{9}{|l|}{ Copayment burden } \\
\hline $\begin{array}{l}\text { Total cost of } \\
\text { prescriptions }\end{array}$ & $\begin{array}{l}\$ 5,278 \\
(\$ 3,808)\end{array}$ & $\begin{array}{l}\$ 4,612 \\
(\$ 3,444)\end{array}$ & $<0.0001$ & $\begin{array}{l}\$ 4,984 \\
(\$ 3,666)\end{array}$ & $\begin{array}{l}\$ 5,075 \\
(\$ 4,580)\end{array}$ & $\begin{array}{l}\$ 4,362 \\
(\$ 5,258)\end{array}$ & $<0.001$ & $\begin{array}{l}\$ 4,783 \\
(\$ 4,88 I)\end{array}$ \\
\hline $\begin{array}{l}\text { Copay per } \\
\text { insulin prescription }\end{array}$ & $\begin{array}{l}\$ 18.63 \\
(\$ 15.42)\end{array}$ & $\begin{array}{l}\$ 20.10 \\
(\$ 18.14)\end{array}$ & $<0.0001$ & $\begin{array}{l}\$ 19.28 \\
(\$ 16.69)\end{array}$ & $\begin{array}{l}\$ 17.28 \\
(\$ 14.28)\end{array}$ & $\begin{array}{l}\$ 18.72 \\
(\$ 16.85)\end{array}$ & 0.017 & $\begin{array}{l}\$ 17.87 \\
(\$ 15.40)\end{array}$ \\
\hline \multicolumn{9}{|l|}{ Comorbidities } \\
\hline $\begin{array}{l}\text { Deyo Charlson } \\
\text { Comorbidity index }\end{array}$ & $1.86(1.46)$ & $1.96(1.60)$ & $<0.001$ & $\begin{array}{l}1.90 \\
(1.52)\end{array}$ & $\begin{array}{l}2.10 \\
(1.72)\end{array}$ & $\begin{array}{l}2.06 \\
(1.74)\end{array}$ & 0.477 & $\begin{array}{l}2.08 \\
(1.73)\end{array}$ \\
\hline
\end{tabular}

(Continued) 
Table 3 (Continued)

\begin{tabular}{|c|c|c|c|c|c|c|c|c|}
\hline & \multicolumn{4}{|c|}{ Basal insulin initiators } & \multicolumn{4}{|c|}{ Insulin mixture initiators } \\
\hline & $\begin{array}{l}\text { Persistent } \\
\mathrm{N}=8,5 \mathrm{I} 5\end{array}$ & $\begin{array}{l}\text { Nonpersistent } \\
N=6,740\end{array}$ & $P$ value & $\begin{array}{l}\text { Total } \\
N=15,255\end{array}$ & $\begin{array}{l}\text { Persistent } \\
N=1,615\end{array}$ & $\begin{array}{l}\text { Nonpersistent } \\
\mathbf{N}=1,117\end{array}$ & $P$ value & $\begin{array}{l}\text { Total } \\
N=2,732\end{array}$ \\
\hline & $\begin{array}{l}\text { Mean \% } \\
\text { (SD) }\end{array}$ & $\begin{array}{l}\text { Mean \% } \\
\text { (SD) }\end{array}$ & & $\begin{array}{l}\text { Mean \% } \\
\text { (SD) }\end{array}$ & $\begin{array}{l}\text { Mean \% } \\
\text { (SD) }\end{array}$ & $\begin{array}{l}\text { Mean \% } \\
(\mathrm{SD})\end{array}$ & & $\begin{array}{l}\text { Mean \% } \\
\text { (SD) }\end{array}$ \\
\hline $\begin{array}{l}\text { Diabetes } \\
\text { complications }\end{array}$ & $20.88 \%$ & $23.09 \%$ & 0.001 & $21.86 \%$ & $24.21 \%$ & $23.19 \%$ & 0.537 & $23.79 \%$ \\
\hline $\begin{array}{l}\text { Macrovascular } \\
\text { complications }\end{array}$ & $48.40 \%$ & $50.50 \%$ & 0.010 & $49.33 \%$ & $51.52 \%$ & $50.94 \%$ & 0.767 & $51.28 \%$ \\
\hline Any mental health & $4.67 \%$ & $5.95 \%$ & $<0.0001$ & $5.24 \%$ & $6.81 \%$ & $6.00 \%$ & 0.396 & $6.48 \%$ \\
\hline \multicolumn{9}{|c|}{ Diabetes-related events } \\
\hline $\begin{array}{l}\text { Inpatient } \\
\text { admissions }\end{array}$ & $0.41 \%$ & $0.89 \%$ & 0.002 & $0.62 \%$ & $0.99 \%$ & $0.54 \%$ & 0.416 & $0.81 \%$ \\
\hline $\begin{array}{l}\text { Mean length } \\
\text { of stay }\end{array}$ & $4.96(3.63)$ & $6.79(6.81)$ & 0.143 & $6.12(5.89)$ & $5.50(6.27)$ & $5.25(3.79)$ & 0.929 & $5.43(5.62)$ \\
\hline ER use & $0.34 \%$ & $0.42 \%$ & 0.566 & $0.37 \%$ & $0.43 \%$ & $0.45 \%$ & 0.956 & $0.44 \%$ \\
\hline \multicolumn{9}{|c|}{ Cardiovascular-related events } \\
\hline $\begin{array}{l}\text { Inpatient } \\
\text { admissions }\end{array}$ & $3.35 \%$ & $4.05 \%$ & 0.233 & $3.66 \%$ & $4.52 \%$ & $4.92 \%$ & 0.786 & $4.69 \%$ \\
\hline $\begin{array}{l}\text { Mean length } \\
\text { of stay }\end{array}$ & $3.28(3.12)$ & $3.83(3.45)$ & 0.050 & 3.55 (3.29) & 3.44 (3.7I) & $4.74(4.64)$ & 0.082 & $4.00(4.17)$ \\
\hline ER use & $1.54 \%$ & $1.99 \%$ & 0.035 & $1.74 \%$ & $2.41 \%$ & $2.15 \%$ & 0.649 & $2.23 \%$ \\
\hline
\end{tabular}

Abbreviations: SD, standard deviation; ER, emergency room.

Across basal insulin and mixture insulin initiators, age, gender, use of human insulin, polypharmacy, and copayment burden were significantly associated with persistence status.

The average number of claims and the average quantity of insulin per day were higher for patients who were persistent. Among basal insulin initiators, patients who were persistent had an average of 9.7 claims compared with an average of 4.7 claims for nonpersistent patients (measure 1). Similarly, persistent patients averaged $0.28 \mathrm{~mL}$ (28 IU) of insulin per day compared with $0.14 \mathrm{~mL}$ of insulin per day for nonpersistent patients (data not shown in table). The same trends observed for measure 2, persistent patients had an average of 7.8 claims and $0.23 \mathrm{~mL}$ (23 IU) per day compared with an average of 3.7 claims and $0.11 \mathrm{~mL}$ (11 IU) per day for nonpersistent patients. Among insulin mixture initiators, the differences were more pronounced: patients without a 90-day gap had an average of 10.7 claims and $0.35 \mathrm{~mL}$ (35 IU) per day in the follow-up period compared with an average of 4.9 claims and $0.16 \mathrm{~mL}$ (16 IU) per day among patients with a 90-day gap during the follow-up period. The difference was also present for measure 2; persistent patients had an average of 9.1 claims and $0.30 \mathrm{~mL}$ (30 IU) per day compared with 3.7 claims and $0.12 \mathrm{~mL}$ (12 IU) per day for nonpersistent patients. The average quantity of insulin per prescription was similar for persistent and nonpersistent patients (basal insulin initiators: $10.8 \mathrm{~mL}$ vs $10.9 \mathrm{~mL}$; insulin mixture initiators: $11.9 \mathrm{~mL}$ vs $11.8 \mathrm{~mL}$ ).
The majority of insulin initiators had a 90-day prescription gap; $73.5 \%$ of basal insulin initiators and $64.0 \%$ of insulin mixture initiators had a 90-day gap in their first year of insulin use. Among these patients with a 90-day gap, the average time from index to the start of the 90-day gap was 80 days ( $\mathrm{SD}=49$ days) for basal insulin initiators and 91 days ( $\mathrm{SD}=60$ days) for insulin mixture initiators. The majority of insulin initiators with a 90-day gap had an insulin prescription following the gap (86.7\% of basal initiators, $81.5 \%$ of insulin mixture initiators). Among these patients with a prescription following a 90-day gap, the average time between the start of the prescription gap and next prescription was 143 days ( $\mathrm{SD}=120$ days) for basal insulin initiators and 146 days ( $\mathrm{SD}=121$ days) for insulin mixture initiators.

\section{Multivariate results: persistence at 12 months (Table 4)}

Basal insulin initiators

By both persistence measures, women were slightly less likely to be persistent than men, with odds ratios (OR) of 0.94 (95\% confidence interval [CI]: 0.87-1.01, $P=0.074$ ) and 0.93 (95\% CI: 0.87-0.99, $P=0.022$ ), respectively. Patients younger than 35 years and those older than 65 years were less likely to be persistent at 12 months by both insulin persistence measures compared with patients aged $45-54$ years. Rural location was positively associated with persistence 
Table 4 Multivariate models predicting persistence

\begin{tabular}{|c|c|c|c|c|}
\hline \multirow[b]{2}{*}{ Sample size } & \multicolumn{4}{|l|}{ Odds ratio $(95 \mathrm{Cl})$} \\
\hline & \multicolumn{2}{|l|}{ Basal cohort $(N=15,255)$} & \multicolumn{2}{|l|}{ Mixture cohort $(\mathrm{N}=\mathbf{2 , 7 3 2 )}$} \\
\hline Variable & $\begin{array}{l}\text { Persistence at } 12 \text { months } \\
\text { measure I }\end{array}$ & $\begin{array}{l}\text { Persistence at } 12 \text { months } \\
\text { measure } 2\end{array}$ & $\begin{array}{l}\text { Persistence at } 12 \text { months } \\
\text { measure I }\end{array}$ & $\begin{array}{l}\text { Persistence at } 12 \\
\text { months measure } 2\end{array}$ \\
\hline Female & $0.94(0.87,1.01)$ & $0.93(0.87,0.99)^{\mathrm{a}}$ & $1.00(0.85,1.17)$ & $0.93(0.80,1.09)$ \\
\hline \multicolumn{5}{|l|}{ Age } \\
\hline$<35$ & $0.63(0.45,0.88)^{\mathrm{a}}$ & $0.66(0.50,0.87)^{\mathrm{b}}$ & $0.76(0.39,1.46)$ & $0.88(0.48,1.60)$ \\
\hline $35-44$ & $0.98(0.85,1.14)$ & $0.90(0.78,1.03)$ & $0.96(0.68,1.35)$ & $0.94(0.68,1.31)$ \\
\hline $55-64$ & $0.92(0.84,1.01)$ & $1.05(0.96,1.14)$ & $0.97(0.78,1.21)$ & I.13 (0.9I, I.39) \\
\hline $65-74$ & $0.69(0.62,0.78)^{c}$ & $0.91(0.82,1.01)$ & $0.97(0.76,1.24)$ & $1.21(0.95,1.54)$ \\
\hline$>74$ & $0.69(0.60,0.79)^{c}$ & $0.77(0.69,0.87)^{c}$ & $0.80(0.60,1.07)$ & $\mathrm{I} .07(0.82, \mathrm{I} .4 \mathrm{I})$ \\
\hline \multicolumn{5}{|l|}{ Region } \\
\hline North Central & $0.98(0.85,1.14)$ & $0.99(0.87,1.13)$ & $0.85(0.6 \mathrm{I}, \mathrm{I} .20)$ & $1.27(0.91,1.77)$ \\
\hline South & $1.07(0.92,1.24)$ & $0.92(0.8 \mathrm{I}, \mathrm{I} .04)$ & $0.84(0.60,1.17)$ & $1.18(0.85,1.63)$ \\
\hline West & $1.17(1.00,1.38)$ & $0.95(0.82,1.09)$ & $0.88(0.6 \mathrm{I}, \mathrm{I} .27)$ & $1.17(0.82,1.68)$ \\
\hline Unknown & $0.75(0.36,1.58)$ & $0.99(0.56,1.77)$ & $0.16(0.02,1.24)$ & $0.60(0.18,1.99)$ \\
\hline Rural & $1.29(1.18,1.41)^{c}$ & $1.11(1.03,1.21)^{\mathrm{a}}$ & I.I2 (0.9।, I.36) & $0.98(0.81,1.19)$ \\
\hline Capitated insurance & $1.26(1.14,1.39)^{c}$ & $1.16(1.06,1.27)^{b}$ & $1.27(1.01,1.59)^{\mathrm{a}}$ & $1.13(0.91,1.41)$ \\
\hline \multicolumn{5}{|l|}{ Index } \\
\hline human & $0.73(0.65,0.83)^{c}$ & $0.70(0.63,0.77)^{c}$ & $0.68(0.58,0.81)^{c}$ & $0.69(0.58,0.81)^{c}$ \\
\hline pen & $0.640 .50,0.82)^{c}$ & $0.78(0.65,0.94)^{b}$ & $0.94(0.79,1.13)$ & $0.99(0.83,1.17)$ \\
\hline Deyo Charlson & $0.99(0.96,1.02)$ & $0.98(0.96,1.01)$ & $0.98(0.93,1.04)$ & $1.00(0.95,1.06)$ \\
\hline \multicolumn{5}{|l|}{ Comorbidity Index } \\
\hline ER admissions & $0.86(0.65,1.15)$ & $0.90(0.7 \mathrm{I}, \mathrm{I} . \mathrm{I} 3)$ & $0.79(0.46,1.34)$ & I.08 $(0.66,1.77)$ \\
\hline Inpatient admissions & $0.86(0.69,1.07)$ & $0.79(0.66,0.95)^{\mathrm{a}}$ & $0.79(0.52,1.19)$ & $0.95(0.64,1.40)$ \\
\hline Diabetes Complications & $1.00(0.90,1.10)$ & $0.94(0.86,1.02)$ & $1.00(0.8 \mathrm{I}, \mathrm{I} .25)$ & $1.03(0.83,1.27)$ \\
\hline Count of OAD agents & $1.02(1.00,1.04)^{\mathrm{a}}$ & $1.02(1.01,1.04)^{b}$ & $1.02(0.98,1.06)$ & $1.02(0.98,1.06)$ \\
\hline Macrovascular complications & $0.94(0.87,1.02)$ & $0.93(0.87,1.00)^{\mathrm{a}}$ & $1.02(0.86,1.22)$ & $0.96(0.81,1.14)$ \\
\hline Mental health disorders & $0.81(0.68,0.96)^{a}$ & $0.79(0.68,0.91)^{b}$ & $0.99(0.7 \mathrm{I}, \mathrm{I} .37)$ & $1.14(0.83,1.57)$ \\
\hline Average copay for insulin & $0.99(0.99,0.99)^{c}$ & $0.99(0.99,1.00)^{c}$ & $0.99(0.99,1.00)^{\mathrm{a}}$ & $0.99(0.99,1.00)^{\mathrm{a}}$ \\
\hline
\end{tabular}

Note: Reference groups: age 45-54; Region: North East; Noncapitated Insurance; a Denotes $P<0.05$; benotes $P<0.0$; ' ${ }^{\mathrm{C} D e n o t e s} P<0.00$ I.

Abbreviations: ER, emergency room; OAD, oral antidiabetic.

at 12 months, with an OR of 1.29 (95\% CI: $1.18-1.41$, $P<0.001)$ for measure 1 and 1.11 (95\% CI: 1.03-1.21, $P=0.01$ ) for measure 2 .

The use of human insulin was significantly associated with poorer persistence (measure 1 : $\mathrm{OR}=0.73$; 95\% CI: $0.65-0.83, P<0.0001$; measure 2: $\mathrm{OR}=0.70$; 95\% CI: $0.63-0.77, P<0.0001)$. Use of insulin pen at index was also associated with poorer persistence by both measures (measure 1: $\mathrm{OR}=0.64 ; 95 \% \mathrm{CI}$ : $0.50-0.82, P<0.0001 ;$ measure $2:$ OR $=0.78 ; 95 \%$ CI: $0.65-0.94, P=0.009)$

Of the clinical variables, the presence of a mental health disorder, which included a diagnosis of depression, was consistently associated with decreased persistence at 12 months by both measures (measure 1: $\mathrm{OR}=0.81$; 95\% CI: $0.68-0.96, P=0.017$; measure 2 : $\mathrm{OR}=0.79$; 95\% CI: $0.68-0.91, P=0.002)$.Increasing the count of oral antidiabetic agent classes was associated with a statistically significant increase in persistence by both measures; however, this increase was modest with an OR of 1.02 for both measures (measure 1: 95\% CI: 1.00-1.04, $P=0.016$; measure 2: 95\% CI: $1.01-1.04, P=0.004$ ). Similarly, increasing the average copayment for insulin by $\$ 1$ decreased the odds of being persistent at 12 months by $1 \%$ for both persistence measures $(P<0.001$ for both measures). Regional location, comorbid burden measured by the Deyo Charlson Comorbidity Index, presence of ER or hospital admissions, and presence of diabetes or macrovascular complications were not statistically significantly associated with persistence.

\section{Insulin mixture initiators}

Among mixed insulin index patients, none of the demographic or clinical characteristics were statistically significantly associated with insulin persistence at 12 months.

The use of human insulin was associated with decreased persistence at 12 months by both measure $1(\mathrm{OR}=0.68$; 
95\% CI: $0.58-0.81, P<0.0001)$ and measure $2(\mathrm{OR}=0.69$; 95\% CI: $0.58-0.81, P<0.0001)$. The use of a pen device was not statistically significantly associated with persistence at 12 months by both measures $(P>0.5)$.

Similar to the basal index insulin patients, the average copayment for insulin was negatively associated with insulin persistence at 12 months, such that a $\$ 1$ increase in average copayment was associated with a $1 \%$ decrease in the odds of persistence at 12 months $(P=0.012$ and $P=0.022$, respectively).

\section{Conclusions}

Insulin use was much lower than expected in the first year following insulin initiation among insulin-naïve patients with type 2 diabetes. Furthermore, the proportion of patients who were persistent with insulin decreased over time, dropping from $83.4 \%$ at 3 months to $67.1 \%$ at 6 months and to $55.8 \%$ at 12 months among basal insulin initiators and dropping from $86.7 \%$ at 3 months to $71.0 \%$ at 6 months and to $59.1 \%$ at 12 months among insulin mixture initiators, as determined by the more lenient of the two measures utilized in this study (ie, measure 2). Using a more conservative gapbased persistence measure (ie, measure 1), 50.6\%, 38.5\%, and $26.5 \%$ of basal insulin initiators were persistent at 3,6 , and 12 months, respectively; $58.5 \%, 47.6 \%$, and $35.0 \%$ of insulin mixture initiators were persistent over the same time periods. Although it is not a measure of discontinuation, the presence of a 90-day gap is an indication of poorer persistence with insulin, particularly, if that gap starts immediately after insulin initiation. Although the majority of patients had a 90-day gap sometime during their first year of insulin use, over one-third of all insulin initiators had a 90-day gap following their first insulin claim.

It is important to distinguish between persistence and permanent medication discontinuation. In this study, persistence was defined as a relatively short-term gap ( 90 days by persistence measure 1) in insulin prescription coverage over a relatively short time period (3, 6, or 12 months). According to Sikka et al, ${ }^{12}$ once patients exceed the permissible gap in prescriptions (90 days in this study), they are no longer considered persistent, even if they have prescription fills following the permissible gap. Over $80 \%$ of patients with an insulin prescription gap of 90 days or greater had additional insulin prescription fills after the gap. Although these patients were nonpersistent by the study definitions, they did not completely discontinue insulin therapy and need further investigation. Persistence measure 2 was developed, in part, as a sensitivity analysis around the gap-based definition of measure 1, allowing for substantially longer intervals between prescriptions to be considered persistent. Even by this lenient measure, only $55 \%-60 \%$ of insulin initiators were persistent at the end of first year.

There is no validated measure available to measure insulin adherence and persistence. The gap period is commonly used as a measure for persistence to oral antidiabetic medications and is well accepted in the literature. Similarly, an early formulation of persistence measure 2 incorporated the current number of claims structure and a requirement of $0.10 \mathrm{~mL}$ index insulin per day, on average. Descriptive analysis of the average daily supply of insulin did not provide a clear, clinically relevant cut off that did not appear arbitrary. Descriptive analysis also indicated that the number of claims requirement largely drove persistence determination. Both of these reasons support excluding insulin quantity per day from persistence determination.

It is possible that patients with prescription gaps of at least 90 days (nonpersistent by measure 1) were stockpiling insulin, potentially causing them to be misclassified as nonpersistent. This did not appear to be the case as patients with prescription gaps of 90 days had significantly lower average insulin supplies per day $(0.14 \mathrm{~mL}$ vs $0.28 \mathrm{~mL}$ among basal insulin initiators).

In spite of these limitations, our results were consistent with other recent research. For example, Cooke et $\mathrm{al}^{13}$ found that $28.7 \%$ of new basal insulin initiators was persistent at 12 months. They also defined persistence as the time (in months) between the first and last insulin fill plus the days supply of the last insulin prescription. This current study was more conservative in that a 90-day gap constituted nonpersistence with insulin. Cooke et al also excluded patients who used any mealtime insulin in the postindex period, matching the exclusion criteria of this current study. Hertz et $\mathrm{al}^{14}$ also conducted a retrospective database analysis and reported that only $37.0 \%$ of new insulin initiators was persistent with insulin therapy at 12 months following index treatment. Similarly, in an evaluation of glargine vs exenatide, Fabunmi et $\mathrm{al}^{15}$ used a 90-day gap to evaluate persistence and determined that less than $60 \%$ of insulin glargine initiators were persistent at 12 months.

Nearly 2,779 insulin users were excluded from this analysis because they only had one prescription of their index insulin. These patients may have required insulin for acute, high hyperglycemia events (ie, they only needed one prescription for insulin, however unlikely that may be). These patients may have also discontinued insulin altogether after one prescription. Future research should examine this 
subgroup of patients to better understand their reasons for discontinuation.

There is evidence that dislike of injections is related to the discontinuing of insulin use, suggesting that the use of insulin pens would be associated with better persistence or adherence than insulin vial and syringe. ${ }^{4}$ Other recent studies have found that insulin pens and vial and syringe have similar adherence profiles. ${ }^{16}$ This current study appears to support the latter standpoint; however, comparing vials and syringe vs insulin pens was not an explicit study objective. Furthermore, the availability of the products may have driven comparisons of insulin type or delivery system. Among basal insulin index patients, the findings regarding delivery system (pen vs vial and syringe) are likely due largely to the unequal distribution of products available; thus, for mixture insulin index patients, index insulin administration was not a significant predictor of persistence.

Multivariate analysis also indicated that human insulin was consistently associated with poorer persistence than analog insulin. This may be due to lower risk of hypoglycemia and greater flexibility in dosing associated with analog insulin. However, provider-related prescribing preferences for analog or human insulin may have also contributed to this finding.

The finding that higher counts of concomitant medications were positively associated with insulin persistence is inconsistent with existing literature. ${ }^{7}$ This association could be due, in part, to the fact that the prescription of additional classes of oral antidiabetic agents is a result of healthcare utilization and monitoring. On the other hand, the presence of comorbid conditions, such as macrovascular complications, was associated with poorer persistence.

While interpreting these results, it is also important to remember limitations of claim databases. Claim data studies are subject to incomplete recording of data or miscoding, particularly with variables that do not drive service payments. These recording errors are most likely to be random errors; therefore, the impact on study results is likely minimal, if any. Further, the data source only includes adjudicated claims, minimizing the risk of data errors and omissions. Similarly, free insulin samples, which are not captured in this dataset, may have caused this study to underestimate insulin use; however, there is no reliable way to estimate the magnitude of this limitation.

Little is known about physician behavior, such as potential channeling of patients to a particular therapy based on information not captured in the claims history. The absence of lab or medical record data further limits the ability of this current study to understand physicians' decision making and prescribing practices. It also limits the ability to evaluate other lifestyle approaches to managing diabetes relative to insulin use and persistence. Furthermore, physician instructions to patients or individual dosing guidelines are unknown. This study only captures prescribed doses that a doctor writes and a patient fills. Finally, because glycemic control was not available in the dataset, we are limited in our ability to interpret results in relation to therapeutic needs of patients, eg, some patients may have discontinued insulin due to high risk of hypoglycemia. It is also possible that a patient may have been prescribed insulin in response to an acute glycemic event, making the patient appear nonpersistent with insulin following the acute insulin episode. Similarly, this current study does not capture how long a patient has had diabetes or the length of diabetes treatment, both of which may play a role in clinical decision making regarding the use of insulin and potentially impact persistence measures 1 and 2 .

In conclusion, in a cohort of patients who were newly initiating insulin, its use was lower than expected in terms of quantity per day, number of claims, and persistence. Consistent predictors of poor persistence with insulin were: use of human insulin at index, age (elderly), presence of mental health disorders, increased average copayment for insulin (although impact was small), and polypharmacy. Although no formal comparisons were conducted, persistence appeared higher for patients starting on mixture insulin compared to basal insulin. Patients who were excluded because they filled only one insulin prescription or used mealtime insulin during the postindex period warrant further investigation.

\section{Disclosures}

Thomson Reuters conducted this study with funding from Eli Lilly and Company. The authors report no conflicts of interest in this work.

\section{References}

1. American Diabetes Association. Economic costs of diabetes in the U.S in 2007. Diabetes Care. 2008;31:596-615.

2. American Diabetes Association. Standards of medical care in diabetes - 2008. Diabetes Care. 2008;31 Suppl 1:S12-S54.

3. Cramer JA. A systematic review of adherence with medications for diabetes. Diabetes Care. 2004;27(5):1218-1224.

4. Catalan VS, Couture JA, Lelorier J. Predictors of persistence of use with the novel antidiabetic agent acarbose. Arch Intern Med. 2001;161: 1106-1112.

5. Dailey G, Kim MS, Lian JF. Patient compliance and persistence with antihyperglycemic drug regimens: evaluation of a medicaid patients population with type 2 diabetes mellitus. Clin Ther. 2001;23:1311-1320.

6. Dezii CM, Kawabata H, Tran M. Effects of once-daily and twice-daily dosing on adherence with prescribed glizipide oral therapy for type 2 diabetes. South Med J. 2002;95:68-71. 
7. Oliveria SA, Menditto LA, Ulcickas Yood M, Koo YH, Wells KE, McCarthy BD. Barriers to the initiation of, and persistence with, insulin therapy. Curr Med Res Opin. 2007;23(12):3105-3112.

8. Paes AH, Bakker A, Soe-Agnie CJ. Impact of dosage frequency on patient compliance. Diabetes Care. 1997;20(10):1512-1517.

9. Odegard PS, Capoccia K. Medication taking and diabetes: a systematic review of the literature. Diabetes Educ. 2007;33(6):1014-1029.

10. Peterson AM, Nau DP, Cramer JA, Benner J, Gwadry-Sridhar F, Nichol M. A checklist for medication compliance and persistence studies using retrospective databases. Value Health. 2007;10(1):3-12.

11. Deyo RA, Cherkin DC, Ciol MA. Adapting a clinical comorbidity index for use with ICD-9-CM administrative databases. J Clin Epidemiol. 1992;45:613-619.

12. Sikka R, Xia F, Aubert RE. Estimating medication persistency using administrative claims data. Am J Manag Care. 2005;11:449-457.
13. Cooke CE, Lee HY, Tong YP, Haines ST. Persistence with injectable antidiabetic agents in members with type 2 diabetes in a commercial managed care organization. Curr Med Res Opin. 2010;26(1):231-238.

14. Hertz RP, Unger AN, Lustik MB. Adherence with pharmacotherapy for type 2 diabetes: a retrospective cohort study of adults with employersponsored health insurance. Clin Ther. 2005;27:1064-1073.

15. Fabunmi R, Nielsen LL, Quimbo R, et al. Patient characteristics, drug adherence patterns, and hypoglycemia costs for patients with type 2 diabetes mellitus newly initiated on exenatide or insulin glargine. Curr Med Res Opin. 2009;25(3):777-786.

16. Pawaskar MD, Camacho FT, Anderson RT, Cobden D, Joshi AV, Balkrishnan R. Health care costs and medication adherence associated with initiation of insulin pen therapy in medicaid-enrolled patients with type 2 diabetes: a retrospective database analysis. Clin Ther. 2007; 29:1294-1305.

\section{Publish your work in this journal}

Patient Preference and Adherence is an international, peer-reviewed, open access journal that focusing on the growing importance of patient preference and adherence throughout the therapeutic continuum. Patient satisfaction, acceptability, quality of life, compliance, persistence and their role in developing new therapeutic modalities and compounds to optimize clinical outcomes for existing disease states are major areas of interest. This journal has been accepted for indexing on PubMed Central. The manuscript management system is completely online and includes a very quick and fair peer-review system. Visit http://www.dovepress.com/ testimonials.php to read real quotes from published authors. 